\title{
Analysis of the association between prior chemotherapy regimens and outcomes of subsequent anti-PD-(L)1 monotherapy in advanced non-small cell lung cancer
}

\author{
Guanghui Gao" ${ }^{1 \#}$ Keyi Jia ${ }^{1 \#}$, Sha Zhao ${ }^{1 \#}$, Xuefei $\mathrm{Li}^{2}$, Chao Zhao ${ }^{2}$, Tao Jiang ${ }^{1}$, Chunxia Su${ }^{1}$, \\ Shengxiang Ren ${ }^{1}$, Fei Zhou ${ }^{1}$, Caicun Zhou ${ }^{1}$ \\ ${ }^{1}$ Department of Medical Oncology, Shanghai Pulmonary Hospital \& Thoracic Cancer Institute, ${ }^{2}$ Department of Lung Cancer and Immunology, \\ Shanghai Pulmonary Hospital, Tongji University School of Medicine, Shanghai 200433, China \\ Contributions: (I) Conception and design: G Gao, S Zhao, K Jia, C Zhou; (II) Administrative support: G Gao, C Zhou; (III) Provision of study \\ materials or patients: G Gao, F Zhou; (IV) Collection and assembly of data: X Li, C Zhao, T Jiang, S Zhao, F Zhou; (V) Data analysis and \\ interpretation: S Zhao, K Jia; (VI) Manuscript writing: All authors; (VII) Final approval of manuscript: All authors. \\ \#These authors contributed equally to this work. \\ Correspondence to: Prof. Caicun Zhou; Fei Zhou. Department of Medical Oncology, Shanghai Pulmonary Hospital \& Thoracic Cancer Institute, \\ Tongji University School of Medicine, No. 507, Zheng Min Road, Shanghai 200433, China. Email: dr_caicunzhou@126.com; drzhoufei@126.com.
}

Background: Immune checkpoint inhibitor (ICI) monotherapy targeting PD-1/PD-L1 has been a prominent option for the patients with advanced non-small cell lung cancer (NSCLC), which is now commonly used in second- or later-line settings after the failure of conventional chemotherapy. Chemotherapy can modulate tumor immunity in drug-dependent manner, suggesting pre-ICI chemotherapeutic regimens might influence the efficacy of immunotherapy. Therefore, it is of interest to investigate the associations between the types of pre-ICI chemotherapy and the outcomes of patients receiving ICIs treatment.

Methods: The data from NSCLC patients who received anti-PD-1/PD-L1 ICI monotherapy after the failure of first-line chemotherapy were retrospectively reviewed. Clinical outcomes of the patients following ICIs monotherapy were compared according to different pre-ICI chemotherapeutic regimens.

Results: Eighty-nine cases receiving ICI monotherapy immediately after the failure of first-line chemotherapy were included into final analysis. The patients in Gem group had the longest PFS (median: $6.50 \mathrm{~m}$ ) following ICIs treatment $(\mathrm{P}=0.031)$, compared to Pem group and Tax group (median: 3.49 and $3.30 \mathrm{~m}$, respectively). Pre-ICI chemotherapy with Gem retained independently associated with favorable PFS ( $\mathrm{P}=0.014$, HR 0.52; 95\% CI, 0.31-0.88) in multivariate analysis after adjusting for other covariates. The patients in Gem group also achieved better objective response rate (ORR) $(\mathrm{P}=0.046)$ and disease control rate (DCR) $(\mathrm{P}=0.005)$ following ICIs treatment compared to those in Pem/Tax group. The differences in depth of response to ICIs between Gem and Pem/Tax groups were also compared. Of the 48 patients who achieved controlled disease and had $\geq 1$ measurable target lesion during ICIs treatment, no greater tumor shrinkage was observed in Gem group ( $\mathrm{P}=0.374)$, however, Gem group trended to have shorter TTM ( $\mathrm{P}=0.074)$.

Conclusions: Prior-line chemotherapy regimens might influence outcomes of the following ICIs monotherapy. Patients received pre-ICI gemcitabine-containing chemotherapy are significantly correlated with longer PFS and better response to ICIs treatment.

Keywords: Chemotherapy; immune checkpoint inhibitors (ICIs); non-small cell lung cancer; programmed cell death factor 1; programmed death ligand 1

Submitted Jul 16, 2019. Accepted for publication Oct 11, 2019.

doi: $10.21037 /$ tlcr.2019.11.25

View this article at: http://dx.doi.org/10.21037/tlcr.2019.11.25 


\section{Introduction}

Among all type of cancers, lung cancer had the highest morbidity and mortality $(1,2)$, with non-small cell lung cancer (NSCLC) being the most common lung cancer pathologic type. In the treatment of advanced NSCLC, the effect of conventional chemotherapy is not satisfying. Targeted therapy could only benefit patients harboring driver mutations which only accounts for part of the patient population $(3,4)$. Moreover, drug resistance remains a main obstacle to prolonging patient survival. Thus, there is an unmet need for novel therapies for treating late-stage NSCLC. Recently, immune checkpoint inhibitor (ICI) monotherapy targeting PD-1/PD-L1 (programmed cell death protein 1/programmed death ligand 1 ) has been a prominent option for the patients with advanced NSCLC, which is now commonly used in second- or later-line settings after the failure of conventional chemotherapy $(5,6)$. However, patients' responses to ICI monotherapy are heterogeneous and the prognostic factors are still not clear enough $(7,8)$. For previously untreated patients, ICIs monotherapy only benefits over chemotherapy for patients with high PD-L1 proportion score, otherwise a combination of ICIs and chemotherapy is more recommended.

Chemotherapy can modulate tumor immunity in drugdependent manner, suggesting pre-ICI chemotherapeutic regimens might influence the efficacy of immunotherapy $(9,10)$. Therefore, it is of interest to investigate the associations between the types of pre-ICI chemotherapy and the outcomes of patients receiving ICIs treatment. There is growing evidence that conventional treatment also has an impact on the tumor microenvironment. For example, combination with anti-angiogenesis therapy promotes the outcome of immunotherapy $(11,12)$. This re-enforces the importance of evaluating the immune status before commencing IO-containing combinations. More than 200 ongoing or planned clinical trials are registered in which IO agents are combined with chemotherapy. However, preclinical data to justify the choice of combinatorial agents is still lacking. What is more, some clinical trials have shown a better outcome when chemotherapy and ICIs are administrated sequentially rather than concomitantly. To sum up, the combinational therapy is still far from perfection.

Gemcitabine is an anti-metabolic chemotherapy agent that is used in treatment of a large variety of malignancies, including breast cancer, ovarian cancer and NSCLC. The strategy of combining immunotherapy with conventional dual drug chemotherapy, among which gemcitabine is recommended equally with other agents, is used in treating advanced NSCLC patients. Considering the various pharmacologic nature of chemotherapy drugs, it is possible that they also interfere in the anti-tumor immune process in different ways and may eventually lead to diverse consequences (13). In this study, we retrospectively analyzed the clinical data collected from a cohort of 89 advanced NSCLC patients, who received ICIs monotherapy after failure in their first-line chemotherapy. We compared the objective response rate (ORR), disease control rate (DCR) and response pattern for ICIs between patients underwent different chemo regiments in prior-line treatment.

\section{Methods}

\section{Study population}

All of the 178 patients with advanced NSCLC who were treated with ICIs monotherapy targeting PD-1/PD-L1 immediately after the failure of prior-line chemotherapy in Shanghai Pulmonary Hospital were retrospectively reviewed. Demographic and clinical variables, mainly including age, sex, histological diagnosis, Eastern Cooperative Oncology Group (ECOG) performance status (PS), mutation status, smoking history and sites of metastasis, were all collected. Smoking status was defined as smokers (including former and current) who had $\geq 100$ cigarettes within their lifetime, and non-smokers were those with a smoking history $<100$ cigarettes in their lifetime. Different types of pre-ICI chemotherapeutic regimens were recorded.

The present study was approved by the ethics committee of Shanghai pulmonary hospital (No. K19-151), Tongji university school of medicine, and written informed consent was obtained from each participant for the collection of clinical data.

\section{Treatment outcomes collection}

Tumor responses were evaluated according to Response Evaluation Criteria in Solid Tumors (RECIST) version 1.1 based on radiographic assessment. We collected the best tumor responses, date of progression and death (or last follow-up) following ICIs treatment from all the patients eligible. To analyze the depth of response, changes in tumor size were estimated as a relative change of the sum of the longest diameters of targeted disease lesions. Maximal 
tumor shrinkage following ICIs treatment was calculated by comparing the greatest tumor reduction with the sum of diameters of target lesions determined at treatment started. The occurrence and grades of immunotherapy-related adverse events (irAEs) were also collected.

\section{Statistical analysis}

All of the statistical analysis was performed using SPSS statistical software (version 22.0; IBM Corporation, Armonk, NY, USA). Baseline clinical pathologic variables were summarized by percentages, and Fisher's exact test or chi-square test was used to assess the distribution of the categorical variables. Progression-free survival (PFS) was calculated from the date of ICIs treatment initiation until the radiographic documentation of objective progression or death, whichever occurred first. Overall survival (OS) was defined from the date of ICIs treatment start to the date of death of any cause or last follow-up in surviving participants. Kruskal-Wallis Test was used to compared the time of chemotherapy between patients with different outcomes. The time to maximal tumor shrinkage (TTM) was defined as the interval since ICIs treatment initiation to the date of maximal tumor shrinkage. PFS and OS curves were estimated via Kaplan-Meier methodology and compared by log-rank test. Cox proportional hazards regression analysis was performed to adjust for treatment effects and baseline prognostic factors. All the variables that might influence PFS and OS were involved in Cox proportional hazard models for univariate analysis, and those with a $\mathrm{P}$ value $<0.1$ identified on univariable analysis were selected for the multivariable analysis. Statistical significance was considered as a two-sided $\mathrm{P}$ value $<0.05$.

\section{Results}

\section{Patient characteristics}

A total of 178 patients underwent ICIs monotherapy between Jan 2016 to Dec 2019 at our institution were reviewed. Among them, 89 cases receiving ICIs treatment as second-line therapy immediately after the failure of firstline chemotherapy were enrolled in present analysis. They were all typical patients with histologically confirmed stage IIIb to IV NSCLC and had no EGFR or $A L K$ genomic aberrations. Baseline clinicopathologic features were summarized in Table S1. All patients were Asian, mainly comprised male (83.1\%), smokers (65.2\%), ECOG PS
$1(96.6 \%)$ and non-squamous (61.8\%). Median age was 64 years, and $52.8 \%$ patients were younger than 65 years. Twelve point four percent of the patients had detectable KRAS I mutation, and $49.4 \%$ had distant metastasis, including brain $(13.5 \%)$ and liver (14.6\%). Most of the included patients (93.3\%) received platinum-based doublet chemotherapy as first-line therapy, with additionally 6 $(6.7 \%)$ of them received single-agent pemetrexed or taxane, and $5(5.6 \%)$ received concurrent anti-angiogenic agents. The median time from chemotherapy to IO initiation was 189 days (maximum 971 days and minimum 23 days). The ICIs used in their second-line therapy are pembrolizumab (34, 38.2\%), nivolumab (20, 22.5\%), tislelizumab (18, $20.2 \%)$, camrelizumab $(9,10.1 \%)$ and sintilimab (2, 2.2\%). According to different pre-ICI chemotherapeutic regimens, the distribution of patients was classified into three groups as follows: gemcitabine-containing (Gem) group (36, $40.45 \%$ ), pemetrexed-containing (Pem) group (38, 42.70\%) and taxane-containing (Tax) group $(15,16.85 \%)$. The main clinicopathologic variables were well balanced across three groups (Table 1). While exceptionally, more smokers were identified in Gem group $(\mathrm{P}=0.010)$ and fewer male patients were identified in Pen group $(\mathrm{P}=0.001)$. In addition, more patients in Pem group were diagnosed with non-squamous histology $(\mathrm{P}=0.000)$.

\section{Survival analysis}

Median follow-up time for survival following ICIs treatment was 7.27 months for total patients and varied from 0.67 to 38.03 months across the three chemotherapeutic groups. At the time of analysis on March 01, 2019, 48 (53.9\%) patients achieved PD or died and 13 (14.6\%) were still on ICIs treatment. Median PFS and OS were 4.37 and 16.83 months, respectively, for the whole patient population. Of note, we found that the patients in GEM group had the longest PFS (median: 6.50 months) following ICIs therapy, with log-rank test showed $\mathrm{P}=0.031$, compared to PEM group and TAX group (median: 3.49 and 3.30 months, respectively), whereas no statistical significance in OS was observed across the three groups (median OS: $16.37,16.83,8.63$ months, respectively) with $\mathrm{P}=0.513$ (Figure 1). The impact of pre-ICI chemotherapy regimens on PFS and OS within individual subgroups of patients was further estimated (Figure S1). As noted, for patients who received ICIs monotherapy as second-line treatment, prior gemcitabine-containing chemotherapy had a consistently positive effect on the survival benefit from ICIs treatment, 
Table 1 Patient demographics and baseline characteristics

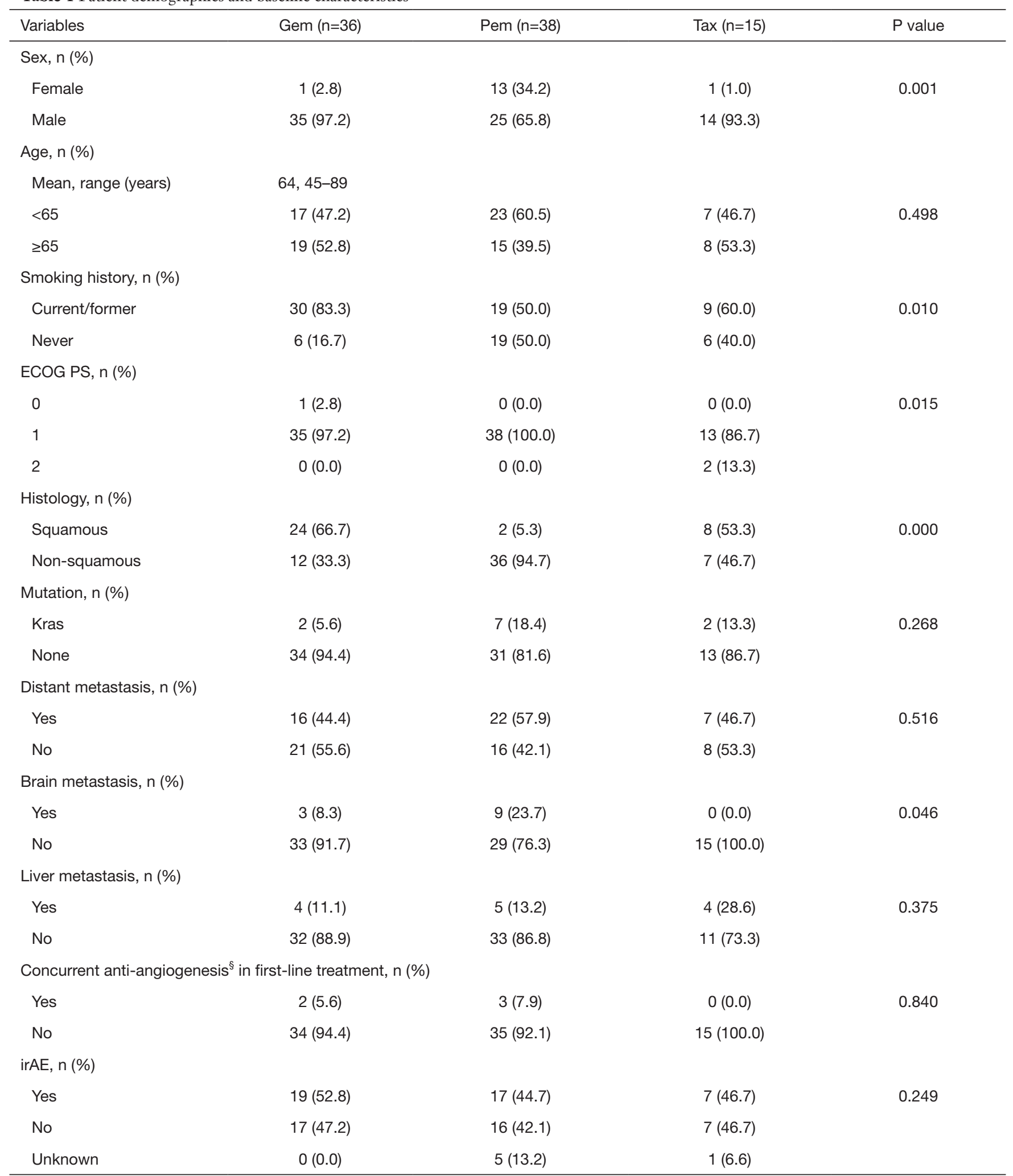

ECOG, Eastern Cooperative Oncology Group; PS, performance status. *, include brain, bone, liver, cervical/abdominal lymph nodes, subcutaneous tissue, adrenal gland; ${ }^{\S}$, include bevacizumab and endostar. 

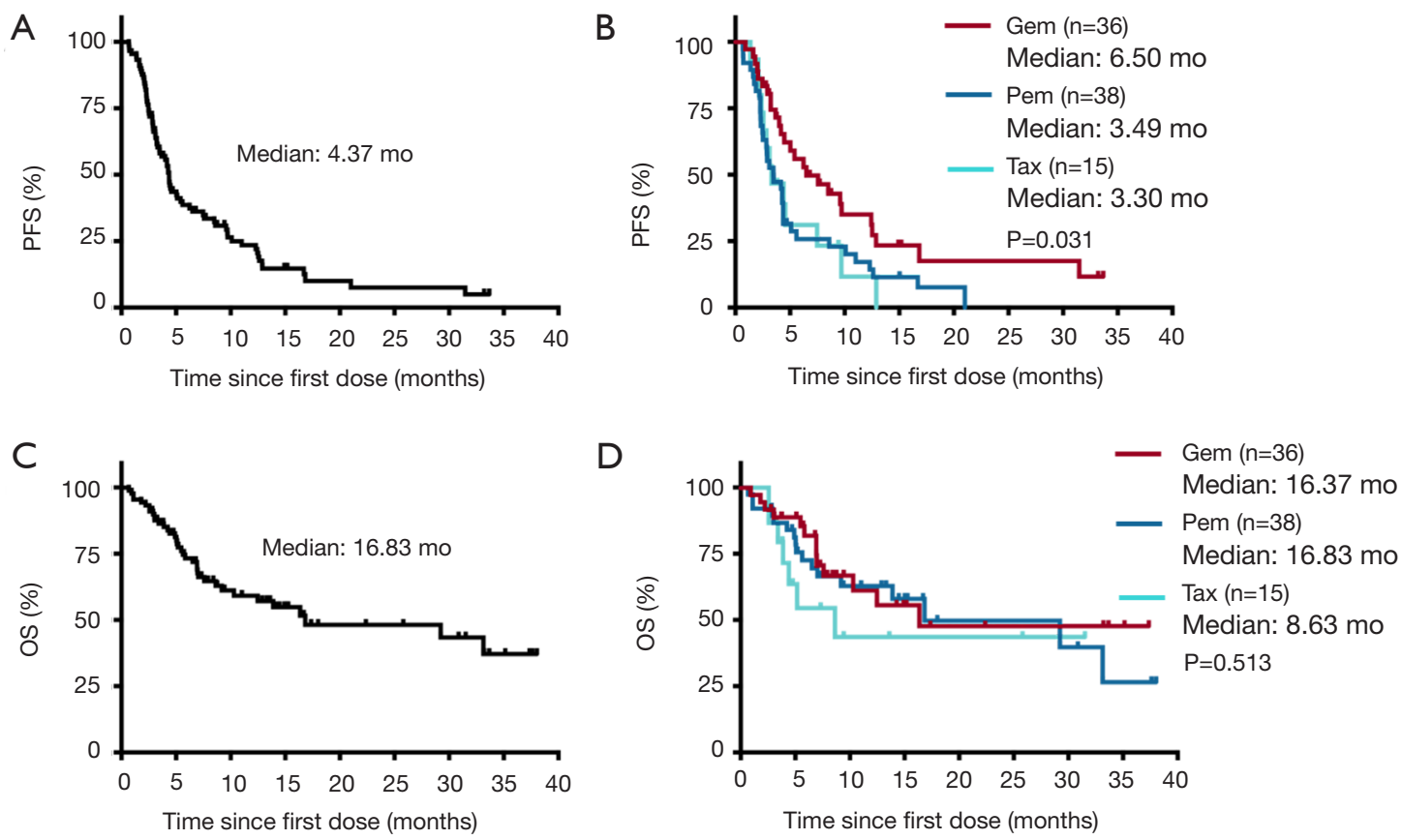

Figure 1 The correlations of survival during ICIs monotherapy and pre-ICI chemotherapy regimens. (A) PFS following ICIs monotherapy of the total patient; (B) PFS following ICIs monotherapy based on different pre-ICI chemotherapeutic regimens; (C) OS following ICIs monotherapy of the total patient; (D) OS following ICIs monotherapy based on different pre-ICI chemotherapeutic regimens. PFS, progression-free survival; OS, overall survival; Gem, gemcitabine; Pem, pemetrexed; Tax, taxane.

with significantly diminished PFS observed in most of the subgroups examined.

The univariable and multivariable analysis for survival was summarized in Tables 2,3. In the univariable analysis for PFS, 4 variables showed $\mathrm{P}<0.1$, including male (HR 0.53; 95\% CI, 0.28-0.98, $\mathrm{P}=0.044$ ), metastasis to brain (HR 3.28; 95\% CI, 1.71-6.32, $\mathrm{P}=0.000$ ), occurrence of irAE (HR 0.67; $95 \%$ CI, $0.42-1.06, \mathrm{P}=0.086)$ and pre-ICI chemotherapy with Gem (HR 0.53; 95\% CI, 0.32-0.86, P=0.010). Multivariable Cox regression demonstrated that pre-ICI chemotherapy with Gem retained independently associated with favorable PFS ( $\mathrm{P}=0.014$, HR 0.52; 95\% CI, 0.31-0.88) after adjusting for other covariates (Table 2). However, in terms of OS, the hazard for death in Gem group was similar compared to Pem/Tax group after adjusting for multiple confounding factors (Table 3).

\section{Response data}

Overall, disease control was achieved in 56 (62.9\%) patients: 18 (20.2\%) had PR and 38 (42.7\%) with SD. The ORR for the total patient cohort was $20.2 \%$. The duration of chemotherapy showed no difference among patients with different outcomes for IO ( $\mathrm{P}=0.229)$. Response data during ICIs treatment are listed in Table 4. As we had observed that pre-ICI chemotherapy with Gem was associated with longer PFS following ICIs treatment, we next dichotomized the patients by pre-ICI chemotherapy regimens into Gem and Pem/Tax groups to analyze the response data. We found that patients in Gem group achieved better ORR $(\mathrm{P}=0.046)$ and DCR $(\mathrm{P}=0.005)$ during ICIs therapy compared with those in Pem/Tax group. We then analyzed the response data separately for smokers and non-smokers. In smokers, we found slightly better DCR in Gem group ( $82.1 \%$ vs. $62.1 \%, \mathrm{P}=0.092$ ). Interestingly, although the sample size is rather small, we did find better ORR in Gem group within non-smokers ( $100 \%$ vs. $46.2 \%, \mathrm{P}=0.017)$. The differences in depth of response to ICIs between Gem and Pem/Tax groups were also compared. Of the patients who achieved controlled disease, 48 had $\geq 1$ measurable target lesion during ICIs treatment. The pooled maximal tumor reduction of the 48 patients were depicted in Figure S2. The median maximal tumor shrinkage was $19.77 \%$ and the median TTM was 2.80 months. No greater 
Table 2 Univariate and multivariate analyses of clinical parameters on PFS

\begin{tabular}{|c|c|c|c|c|}
\hline Parameters & \multicolumn{2}{|c|}{ Univariate analysis } & \multicolumn{2}{|c|}{ Multivariate analysis } \\
\hline Age, year (<65 vs. $\geq 65)$ & $1.15(0.72-1.82)$ & 0.563 & - & - \\
\hline Gender (female vs. male) & $0.53(0.28-0.98)$ & 0.044 & $0.83(0.42-1.62)$ & 0.825 \\
\hline Smoking (never vs. smoker) & $0.68(0.42-1.10)$ & 0.119 & - & - \\
\hline Mutation (none vs. Kras) & $1.61(0.82-3.15)$ & 0.163 & - & - \\
\hline Brain metastasis (no vs. yes) & $3.28(1.71-6.32)$ & 0.000 & $3.31(1.69-6.48)$ & 0.000 \\
\hline Liver metastasis (no vs. yes) & $1.54(0.82-2.88)$ & 0.181 & - & - \\
\hline irAE (no/unknown vs. yes) & $0.67(0.42-1.06)$ & 0.086 & $0.67(0.42-1.06)$ & 0.087 \\
\hline
\end{tabular}

PFS, progression-free survival; HR, hazard rate; ECOG, Eastern Cooperative Oncology Group; PS, performance status; NSq, nonsquamous; Sq, squamous; irAEs, immune-related adverse events.

Table 3 Univariate and multivariate analyses of clinical parameters on OS

\begin{tabular}{|c|c|c|c|c|}
\hline Parameters & \multicolumn{2}{|c|}{ Univariate analysis } & \multicolumn{2}{|c|}{ Multivariate analysis } \\
\hline Age, year (<65 vs. $\geq 65)$ & $1.55(0.80-2.99)$ & 0.190 & - & - \\
\hline Gender (female vs. male) & $0.96(0.40-2.31)$ & 0.931 & - & - \\
\hline Smoking (never vs. smoker) & $1.05(0.52-2.13)$ & 0.899 & - & - \\
\hline Mutation (none vs. Kras) & $1.74(0.76-3.96)$ & 0.190 & - & - \\
\hline Brain metastasis (no vs. yes) & $3.51(1.54-8.01)$ & 0.003 & $6.37(2.40-16.90)$ & 0.000 \\
\hline Liver metastasis (no vs. yes) & $1.71(0.75-3.92)$ & 0.250 & - & - \\
\hline irAE (no/unknown vs. yes) & $0.76(0.39-1.45)$ & 0.399 & - & - \\
\hline
\end{tabular}

PFS, progression-free survival; HR, hazard rate; ECOG, Eastern Cooperative Oncology Group; PS, performance status; NSq, nonsquamous; Sq, squamous; irAEs, immune-related adverse events.

tumor shrinkage was observed in Gem group $(\mathrm{P}=0.374)$, however, Gem group trended to have shorter TTM $(\mathrm{P}=0.074)$ (Table 5).

\section{Discussion}

In this study, our data showed that lung cancer patients received different prior-line chemotherapy regimens might display diverse outcomes for subsequent ICIs monotherapy. Patients received gemcitabine-containing chemotherapy as their first-line treatment were more likely to have longer PFS with following ICIs treatment. Additionally, we also detected different response patterns between gemcitabine group and pemetrexed/taxane group. Patients underwent prior gemcitabine-containing chemotherapy trended to reach maximal tumor shrinkage earlier in their second-line 
Table 4 Data for treatment response

\begin{tabular}{|c|c|c|c|c|}
\hline Parameters & Total $(\mathrm{N}=89)$ & Gem (n=36) & Pem/Tax $(n=53)$ & $P$ value \\
\hline PR & $18(20.2)$ & $11(30.6)$ & 7 (13.2) & \\
\hline SD & $38(42.7)$ & $18(50.0)$ & $20(37.7)$ & \\
\hline PD & $33(37.1)$ & $7(19.4)$ & $26(49.1)$ & \\
\hline DCR, \% & 62.9 & 80.6 & 50.9 & 0.005 \\
\hline
\end{tabular}

PR, partial response; SD, stable disease; PD, progression disease; ORR, objective response rate; DCR, disease control rate.

Table 5 Data for tumor response during ICIs treatment

\begin{tabular}{|c|c|c|c|c|}
\hline Parameters & Total $(n=48)$ & Gem $(n=25)$ & Pem/Tax $(n=23)$ & $P$ value \\
\hline \multicolumn{5}{|l|}{ Maximal tumor shrinkage } \\
\hline High, n (\%) & $24(50.0)$ & $14(56.0)$ & $10(56.5)$ & 0.386 \\
\hline Low, n (\%) & $24(50.0)$ & $11(44.0)$ & $13(43.5)$ & \\
\hline
\end{tabular}

TTM, time to maximal tumor shrinkage.

ICIs treatment, comparing with those had pemetrexed/ taxane chemotherapy regimens.

A number of clinical trials have proved that the combination of chemotherapy or anti-angiogenesis therapy with immunotherapy could improve treatment response rate $(9,10,14,15)$. However, the sequential use of other anti-cancer therapy and immunotherapy has rarely been studied. Nakahama et al. (16) reported a retrospective study in 2017. In their research, they found that patient received bevacizumab as part of their pre-nivolumab treatment tended to have higher response rate to secondline nivolumab. They also discovered better DCR for nivolumab in patients who reached disease control in their prior-line treatment, which included bevacizumab, platinum-based doublets chemotherapy and tyrosine kinase inhibitor, though no difference was found within these arms. Given the fact that chemotherapy alone could notably alter immune microenvironment and their varied pharmacological nature, it is reasonable to infer that different chemo agent may lead to different response to immunotherapy. As far as we know, this is the first study that found survival differences for second-line ICIs within groups went through different chemotherapies.

In the treatment of advanced lung cancer, first-line
ICIs monotherapy only benefited over chemotherapy for patients with high PD-L1 tumor proportion score $(7,8)$. For unselected patients, accumulating clinical evidences have shown that the addition of ICIs to standard platinumcontaining dual drug chemotherapy could notably increase tumor response and prolong patient survival $(9,10,17)$. The mechanism of this synergetic effect of chemotherapy and immunotherapy is rather complicated. Theoretically, it was generally accepted that chemotherapy agents could enhance anti-tumor immunity by damaging tumor cells and release more tumor antigens, including tumor-specific antigen (TSA) and tumor-associated antigen (TAA). However, in clinical settings, there were no data indicating that enhancing tumor antigenicity could directly improve prognosis. In fact, conventional chemotherapy drugs could interfere in almost all phases of the immune response against malignancy (13). For example, paclitaxel could induce costimulatory molecule expression and cytokine release by dendritic cells (18) increasing intertumoral infiltration of $\mathrm{T}$ cell (19) and diminish the immunosuppressive activity of myeloid-derived suppressor cells (MDSCs) in tumor (20). Referring to previous researches, we found that gemcitabine had a much wider effect on anti-tumor immunity, which was in accordance with our finding in current study. 
Gemcitabine-containing chemotherapy could resurrect the defective cross-presentation function of tumor infiltrating DCs (21), which was essential for the maturation of CD8 ${ }^{+}$ $\mathrm{T}$ cells in tumor situ (22). Moreover, the suppression of MDSCs caused by gemcitabine treatment was found in multiple tumor types (23-25). In the initial phase of antitumor immune response, exposing to gemcitabine led to upregulation of human leucocyte antigen class 1 (HLA1) which enhanced the antigenicity of tumors (26). All these researches together showed us that gemcitabine has a more comprehensive effect on immune microenvironment than other chemotherapy agents, may provide possible explanations to what we observed in our study.

The limitation of our study is obvious, including its retrospective nature. We did observe higher ORR and DCR for second-line immunotherapy in patients received gemcitabine in prior-line. Difference between groups was found in PFS but not OS, indicating the possibility of selection bias. However, without any immune monitoring, we cannot elucidate the mechanisms behind the phenomena we observed, but to offer possible explanations from published data. Further research aimed on gemcitabine's unique effect to anti-tumor immunity, such as its high efficacy against MDSCs, is certainly needed. Since smokers were more prevalent in gemcitabine group, the diverse response could potentially be attributable to differed antigen levels. Finally, the study was based on a small number of patients from one single institution. To reconfirm the authenticity of these findings, randomized research based on larger cohort is required.

To sum up, the ORR and DCR of second-line immune therapy was higher in patients underwent first-line gemcitabine-containing chemotherapy than pemetrexed or taxane chemotherapy regiments. Tumor exposed to gemcitabine at past displayed quicker response to following ICIs monotherapy than those who had pemetrexed or taxane for their the first-line. Further investigations are expected to determine whether the use of gemcitabine in the first-line could improve the prognosis of NSCLC patients comparing to other chemo agents.

\section{Acknowledgments}

Funding: This study was supported by grants from the National Natural Science Foundation of China (No. 81672286, No. 81871865 and No. 81874036), the Innovation Program for Basic Research of the Science and Technology Commission Shanghai Municipality
(No. 16JC1405900), the National Key Research and Development Projects of China (No. 2016YFC0902300).

\section{Footnote}

Conflicts of Interest: The authors have no conflicts of interest to declare.

Ethical Statement: The authors are accountable for all aspects of the work in ensuring that questions related to the accuracy or integrity of any part of the work are appropriately investigated and resolved. The present study was approved by the ethics committee of Shanghai pulmonary hospital (No. K19-151), Tongji university school of medicine, and written informed consent was obtained from each participant for the collection of clinical data.

\section{References}

1. Chen W, Zheng R, Zhang S, et al. Lung cancer incidence and mortality in China, 2009. Thorac Cancer 2013;4:102-8.

2. Siegel RL, Miller KD, Jemal A. Cancer statistics, 2019. CA Cancer J Clin 2019;69:7-34.

3. Pirker R, Buder A, Filipits M. Osimertinib in advanced EGFR T790M-positive non-small-cell lung cancer: the clinical impact of AURA3. Transl Cancer Res 2017;6:S265-9.

4. Zhou C, Wu YL, Chen G, et al. Final overall survival results from a randomised, phase III study of erlotinib versus chemotherapy as first-line treatment of EGFR mutation-positive advanced non-small-cell lung cancer (OPTIMAL, CTONG-0802). Ann Oncol 2015;26:1877-83.

5. Rittmeyer A, Barlesi F, Waterkamp D, et al. Atezolizumab versus docetaxel in patients with previously treated non-small-cell lung cancer (OAK): a phase 3, openlabel, multicentre randomised controlled trial. Lancet 2017;389:255-65.

6. Ettinger DS, Aisner DL, Wood DE, et al. NCCN Guidelines Insights: Non-Small Cell Lung Cancer, Version 5.2018. J Natl Compr Canc Netw 2018;16:807-21.

7. Mok TSK, Wu YL, Kudaba I, et al. Pembrolizumab versus chemotherapy for previously untreated, PD-L1expressing, locally advanced or metastatic non-small-cell lung cancer (KEYNOTE-042): a randomised, open-label, controlled, phase 3 trial. Lancet 2019;393:1819-30. 
8. Reck M, Rodriguez-Abreu D, Robinson AG, et al. Updated Analysis of KEYNOTE-024: Pembrolizumab Versus Platinum-Based Chemotherapy for Advanced NonSmall-Cell Lung Cancer With PD-L1 Tumor Proportion Score of 50\% or Greater. J Clin Oncol 2019;37:537-46.

9. Gandhi L, Rodriguez-Abreu D, Gadgeel S, et al. Pembrolizumab plus Chemotherapy in Metastatic NonSmall-Cell Lung Cancer. N Engl J Med 2018;378:2078-92.

10. Paz-Ares L, Luft A, Vicente D, et al. Pembrolizumab plus Chemotherapy for Squamous Non-Small-Cell Lung Cancer. N Engl J Med 2018;379:2040-51.

11. Manegold C, Dingemans AC, Gray JE, et al. The Potential of Combined Immunotherapy and Antiangiogenesis for the Synergistic Treatment of Advanced NSCLC. J Thorac Oncol 2017;12:194-207.

12. Zhao S, Ren S, Jiang T, et al. Low-Dose Apatinib Optimizes Tumor Microenvironment and Potentiates Antitumor Effect of PD-1/PD-L1 Blockade in Lung Cancer. Cancer Immunol Res 2019;7:630-43.

13. Galluzzi L, Zitvogel L, Kroemer G. Immunological Mechanisms Underneath the Efficacy of Cancer Therapy. Cancer Immunol Res 2016;4:895-902.

14. Carbone DP, Reck M, Paz-Ares L, et al. First-Line Nivolumab in Stage IV or Recurrent Non-Small-Cell Lung Cancer. N Engl J Med 2017;376:2415-26.

15. Socinski MA, Jotte RM, Cappuzzo F, et al. Atezolizumab for First-Line Treatment of Metastatic Nonsquamous NSCLC. N Engl J Med 2018;378:2288-301.

16. Nakahama K, Isa SI, Tamiya A, et al. The Association Between Chemotherapy Immediately Before Nivolumab and Outcomes Thereafter. Anticancer Res 2017;37:5885-91.

17. High TMB Predicts Immunotherapy Benefit. Cancer Discov 2018;8:668.

18. Pfannenstiel LW, Lam SS, Emens LA, et al. Paclitaxel enhances early dendritic cell maturation and function

Cite this article as: Gao G, Jia K, Zhao S, Li X, Zhao C, Jiang T, Su C, Ren S, Zhou F, Zhou C. Analysis of the association between prior chemotherapy regimens and outcomes of subsequent anti-PD-(L)1 monotherapy in advanced non-small cell lung cancer. Transl Lung Cancer Res 2019;8(6):920-928. doi: $10.21037 /$ tlcr.2019.11.25 through TLR4 signaling in mice. Cell Immunol 2010;263:79-87.

19. Demaria S, Volm MD, Shapiro RL, et al. Development of tumor-infiltrating lymphocytes in breast cancer after neoadjuvant paclitaxel chemotherapy. Clin Cancer Res 2001;7:3025-30.

20. Sevko A, Michels T, Vrohlings M, et al. Antitumor effect of paclitaxel is mediated by inhibition of myeloidderived suppressor cells and chronic inflammation in the spontaneous melanoma model. J Immunol 2013;190:2464-71.

21. McDonnell AM, Lesterhuis WJ, Khong A, et al. Tumorinfiltrating dendritic cells exhibit defective crosspresentation of tumor antigens, but is reversed by chemotherapy. Eur J Immunol 2015;45:49-59.

22. Thompson ED, Enriquez HL, Fu YX, et al. Tumor masses support naive $\mathrm{T}$ cell infiltration, activation, and differentiation into effectors. J Exp Med 2010;207:1791-804.

23. Suzuki E, Kapoor V, Jassar AS, et al. Gemcitabine selectively eliminates splenic Gr-1+/CD11b+ myeloid suppressor cells in tumor-bearing animals and enhances antitumor immune activity. Clin Cancer Res 2005;11:6713-21.

24. Mundy-Bosse BL, Lesinski GB, Jaime-Ramirez AC, et al. Myeloid-derived suppressor cell inhibition of the IFN response in tumor-bearing mice. Cancer Res 2011;71:5101-10.

25. Eriksson E, Wenthe J, Irenaeus S, et al. Gemcitabine reduces MDSCs, tregs and TGFbeta-1 while restoring the teff/treg ratio in patients with pancreatic cancer. J Transl Med 2016;14:282.

26. Liu WM, Fowler DW, Smith P, et al. Pre-treatment with chemotherapy can enhance the antigenicity and immunogenicity of tumours by promoting adaptive immune responses. Br J Cancer 2010;102:115-23. 


\section{Supplementary}

A

PFS

All patients $(\mathrm{N}=89)$

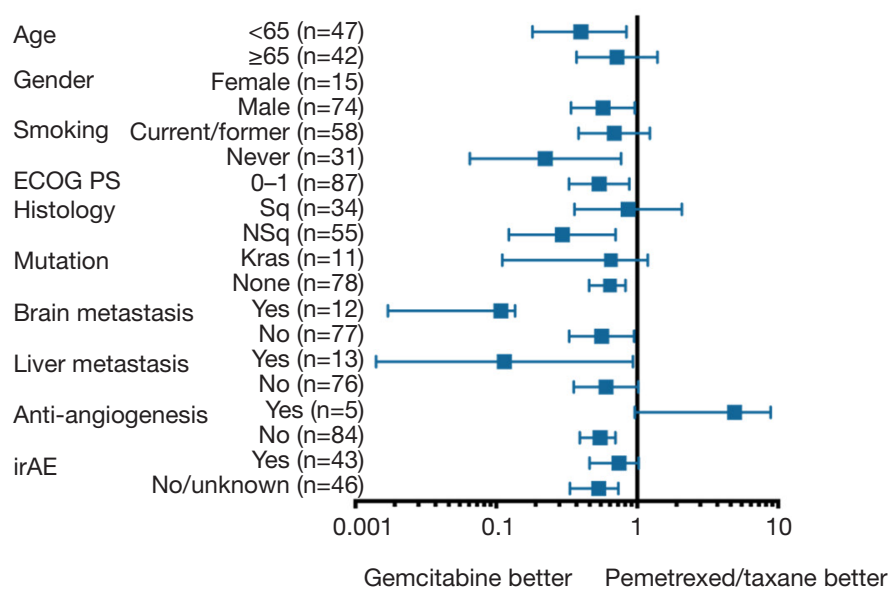

B

OS

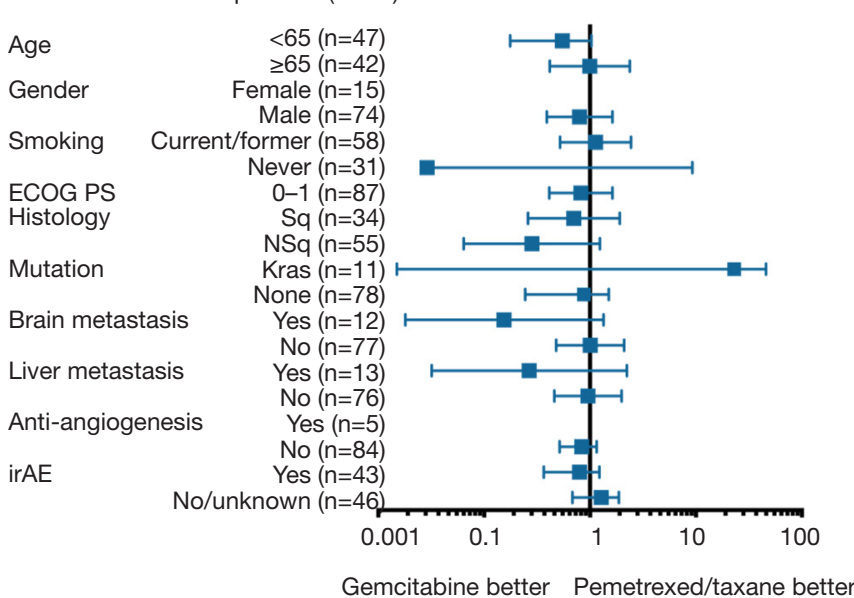

Figure S1 Forest plot of subgroup analysis by baseline characteristics for PFS (A) and OS (B) following ICIs monotherapy. PFS, progression-free survival; OS, overall survival; ECOG, Eastern Cooperative Oncology Group; PS, performance status; irAE, immunerelated adverse event. 
Table S1 Patient demographics and baseline characteristics

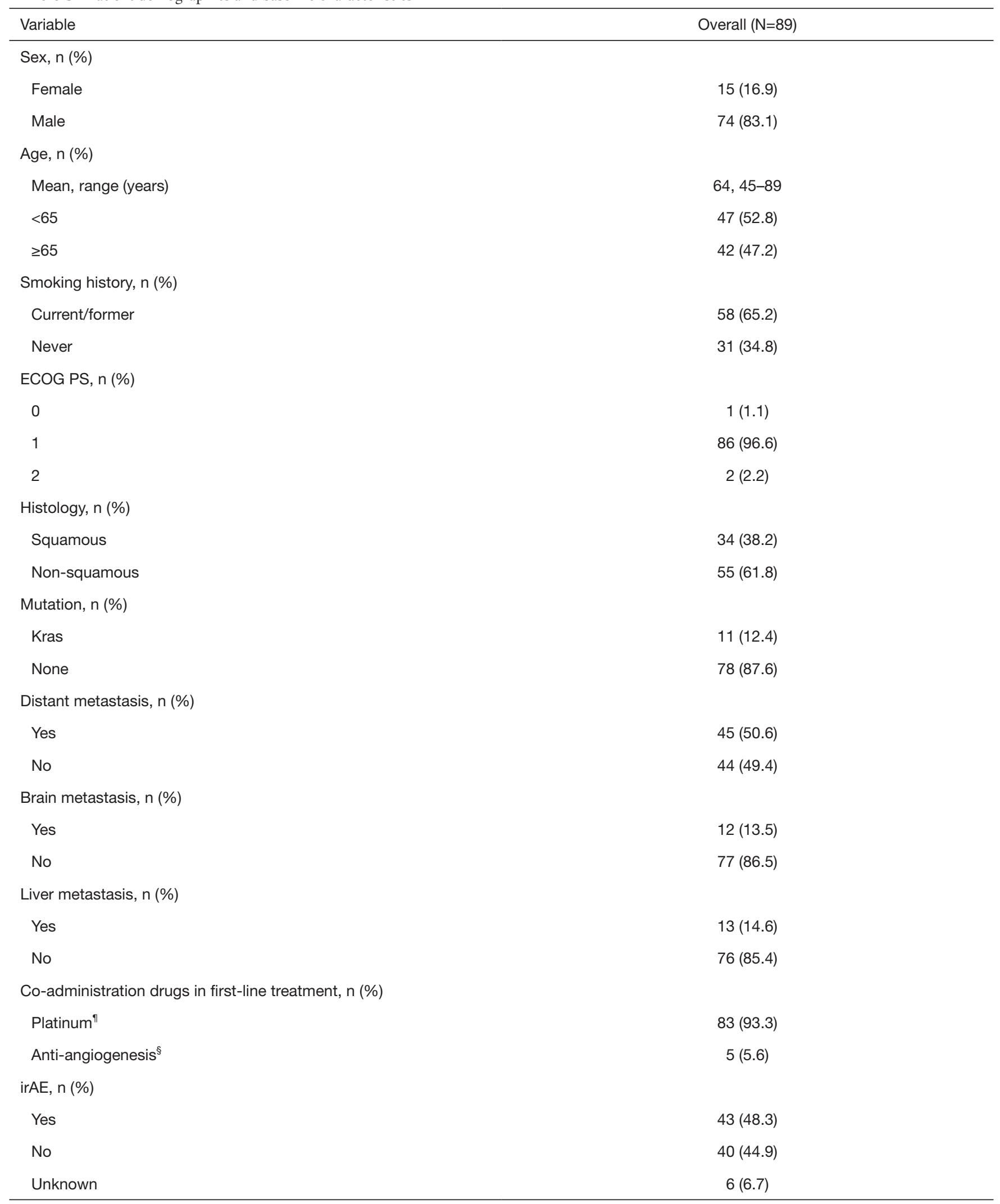

ECOG, Eastern Cooperative Oncology Group; PS, performance status. *, include brain, bone, liver, cervical/abdominal lymph nodes, subcutaneous tissue, adrenal gland; ๆ , include paclitaxel, nab-paclitaxel; ${ }^{\S}$, include bevacizumab and endostar. 


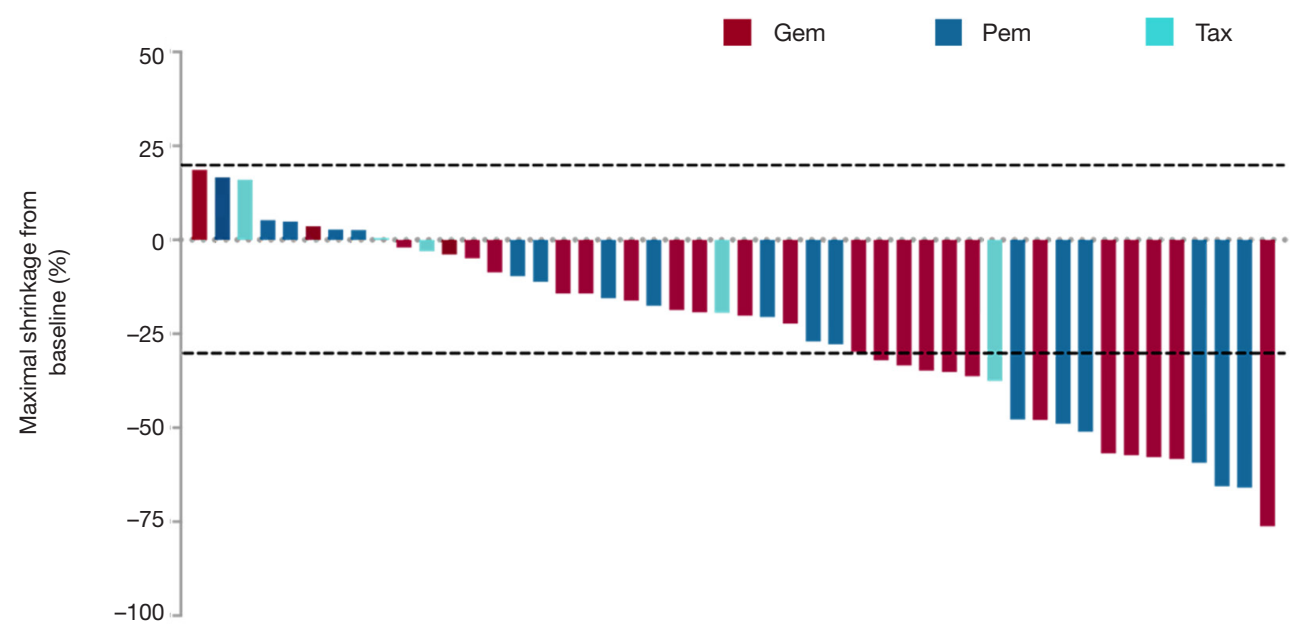

Figure S2 Maximal Tumor Shrinkage in the 48 patients who achieved controlled disease and had $\geq 1$ measurable target lesion during ICIs treatment. Gem, gemcitabine; Pem, pemetrexed; Tax, taxane. 